\title{
OPEN Traditional African remedies induce hemolysis in a glucose-6-phopshate dehydrogenase deficient zebrafish model
}

\author{
Olufunmilayo Arogbokun ${ }^{1}$, Margaret Shevik ${ }^{2}$, Tina Slusher ${ }^{3,4}$, Zubaida Farouk ${ }^{5}$, \\ Alexis Elfstrum ${ }^{2}$, Jenna Weber ${ }^{6}$, Sarah E. Cusick ${ }^{7} \&$ Troy Lund $^{2 \bowtie}$
}

Traditional remedies are widely used throughout Africa in routine care for infants. However, such remedies could have detrimental effects. Acute bilirubin encephalopathy (ABE) and kernicterus spectrum disorder (KSD) are common newborn health conditions in the developing world, contributing to substantial neonatal mortality and morbidity. They frequently occur in children with glucose-6-phopshate dehydrogenase (G6PD) deficiency. Using our established zebrafish model of G6PD deficiency, we tested the effects of three traditional compounds used in the care of the newborn umbilical cord: eucalyptus oil, methylated spirits, and Yoruba herbal tea. We found that eucalyptus oil induced a $13.4 \%$ increase in a hemolytic phenotype versus control, while methylated spirits showed a $39.7 \%$ increase in affected phenotype. Yoruba herbal tea exposure showed no effect. While methylated spirits are already a known pro-oxidant, these data indicate that eucalyptus oil may also be a hemolytic trigger in those with G6PD deficiency. Discovering which agents may contribute to the pathophysiology of G6PD deficiency is critical to eliminate ABE and KSD, especially in countries with a high prevalence of G6PD deficiency. The next step in elucidating the role of these agents is to determine the clinical correlation between the use of these agents and ABE/KSD.

The World Health Organization estimates that up to $80 \%$ of Africa uses traditional medicine for primary health care $^{1}$, but the efficacy and safety of these treatments has not yet been scientifically established. Within Nigeria, traditional remedies are used prominently ${ }^{2}$. The Roll Back Malaria Partnership recently found that the first choice of treatment for high fever in Nigerian children is an herbal treatment ${ }^{3}$. Other studies have shown that mothers commonly use these traditional remedies for their neonates, both for treatment and for routine care ${ }^{1,4}$. The remedies used are wide-ranging and include herbs, spices, and other local vegetative species.

Glucose-6-phosphate dehydrogenase (G6PD) deficiency is the most common X-linked genetic disease in the world with up to 400 million individuals affected. G6PD is the first step in the pentose phosphate shunt to product the important reducing unit NADPH. NADPH provides cellular protection against oxidative stress during cellular metabolism and also from external pro-oxidant exposure. While most cells have redundant cellular mechanisms to deal with oxidative stress, red blood cells rely solely on NADPH produced by G6PD, as they have no mitochondria nor nucleus. G6PD deficiency offers some protection against several malarial infections and is found in up to $30 \%$ of Africa populations depending on the region ${ }^{5-7}$. Unfortunately, many of the drugs used to treat malaria are strong pro-oxidants-with primaquine being the prototypical example-and cause druginduced hemolysis in affected individuals.

African neonates have immature antioxidant cellular metabolism and are at increased risk of hyperbilirubinemia and kernicterus with and without acute hemolysis. Tight associations have been demonstrated between G6PD deficiency and jaundiced African neonates ${ }^{8-12}$.

Some traditional remedies such as mentholated rub, camphor, naphthalene, and henna have been studied and shown to cause hemolysis in neonates with G6PD deficiency ${ }^{13-15}$. We have observed the use of eucalyptus

\footnotetext{
${ }^{1}$ The University of North Carolina at Chapel Hill, Chapel Hill, NC, USA. ${ }^{2}$ Pediatric Blood and Marrow Transplant Program, Global Pediatrics, University of Minnesota, MMC 366, 420 Delaware St SE, Minneapolis, MN 55455, USA. ${ }^{3}$ Department of Pediatrics, Hennepin Healthcare University of Minnesota, Minneapolis, USA. ${ }^{4}$ Bowen University of Teaching Hospital, Ogbomoso, Nigeria. ${ }^{5}$ Department of Paediatrics, Aminu Kano Teaching Hospital, Bayero University, Kano, Nigeria. ${ }^{6}$ Utah School of Medicine, Salt Lake City, UT, USA. ${ }^{7}$ Division of Epidemiology, Department of Pediatrics, University of Minnesota, Minneapolis, USA. ${ }^{\circledR}$ email: Iundx072@umn.edu
} 
A

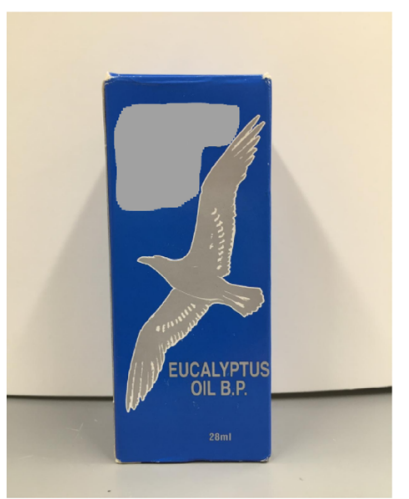

Eucalyptus Oil
B

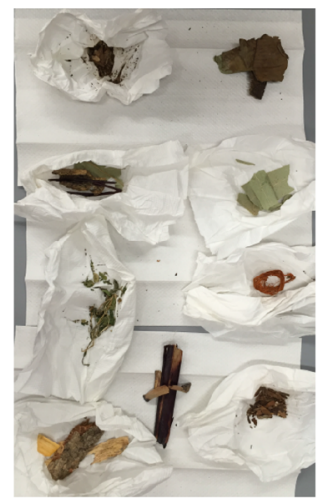

"Yoruba Tea"

C

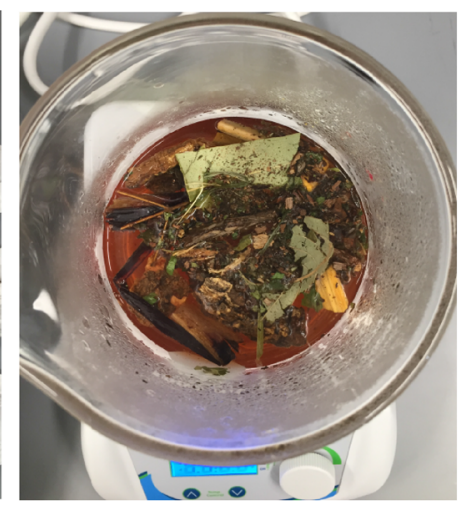

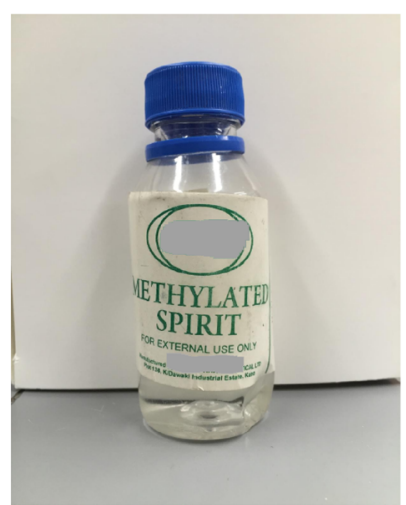

Methylated Spirit

Figure 1. Compound tested in the G6PD-deficient zebrafish model. (A) Eucalyptus oil. (B) A variety of roots, bark, herbs to brew Yoruba tea (right panel). (C) Methylated spirits.

oil, methylated spirits, and traditional Yoruba tea for neonatal umbilical cord care as well as for general "healing properties." Many of these infants going on to develop jaundice. With Nigeria having a high burden of G6PD deficiency ${ }^{16}$, large traditional remedy usage, and the recent prioritization of identification of effective treatments of neonatal jaundice by Nigerian pediatricians, it is important to explore the safety and impact of such treatments ${ }^{12,17}$.

We aimed to study the impacts of these widely used traditional remedies in our previously established G6PDdeficient zebrafish ${ }^{18}$. This model utilizes morpholino-induced downregulation of G6PD (at the 1-2 cell stage) followed by compound exposure. We have shown that this model produces a hemolytic anemia which generates a clear phenotype consisting of robust pericardial edema (and often anasarca, see Supplementary Figure 1). This system allowed rapid high throughput testing of compounds and has been shown to be very responsive to pro-oxidant exposures in producing the expected phenotype ${ }^{18}$.

\section{Results}

To investigate oxidative stress induced hemolysis, we tested eucalyptus oil, methylated spirit, and Yoruba tea in our G6PD-deficient zebrafish mode ${ }^{18}$ (Fig. 1). Initial experiments to establish appropriate dilutions which did not cause high levels of toxicity or massive death were performed in wildtype animals.

G6PD deficiency was induced through the use of morpholino (MO) injections at the 1-2 cell stage as previously described ${ }^{18}$. At $24 \mathrm{~h}$ post-fertilization (hpf), embryos were dechorionated and exposure to selected compounds. After $48 \mathrm{~h}$ of compound exposure, animals were tallied for those with an edematous phenotype and those with a wild-type appearance (Fig. 2). Comparison of animals from uninjected, G6PD MO, and Random MO injected groups showed a low-level of hemolysis from injection alone, a known possible phenomenon when injection of foreign nucleic acid induces a stress response $\mathrm{e}^{18}$.

We first tested eucalyptus oil diluted in fishwater and showed a significant loss of normal phenotype in G6PD morphants exposed to eucalyptus compared to non-exposure phenotype (38.5\% normal versus $61.0 \%$ normal, respectively, Fig. 3A). As with our prior report, death is most often accompanied by a hemolytic phenotype ${ }^{18}$. Consequently, $61.5 \%$ of the G6PD morphants exposed to eucalyptus displayed hemolytic/dead phenotype compared to non-exposure animals at $39.0 \%$ (Fig. 3A).

We next tested methylated spirits and showed a significant loss of normal phenotype in G6PD morphants exposed to methylated spirits compared to non-exposure phenotype ( $15.3 \%$ normal versus $48.5 \%$ normal, respectively, Fig. 3B). Consequently, $84.7 \%$ of the G6PD morphants exposed to methylated spirits displayed hemolytic/ dead phenotype compared to non-exposure animals at 51.5\% (Fig. 3B). It should be noted that a clutch can experience a greater level of death at baseline, as these animals are very outbred, frequently giving rise to heterogeneity in offspring. In this clutch, the G6PD MO death rate was $42.3 \%$.

Finally, Yoruba tea was made by boiling local ingredients for $20 \mathrm{~min}$ in fishwater. The tea was then diluted in fresh fishwater. The animals in the Yoruba tea environment showed some loss of normal phenotype in G6PD morphants compared to non-exposure phenotype (69\% normal versus 53\% normal, respectively, Fig. 3C). Consequently, $47 \%$ of the G6PD morphants exposed to Yoruba tea displayed hemolytic/dead phenotype compared to non-exposure animals at $31 \%$ (Fig. 3C).

Although the data show a propensity for increased hemolysis in the eucalyptus and methylated spirit exposed G6PD morphants, there is always clutch-to-clutch variation and background phenotype generated from both compound exposure and MO injection. Therefore, in the unexposed morphants, we normalized by phenotype to produce a treatment effect and compared Random MO and G6PD MO injected animals. Figure 4 shows that eucalyptus oil produced a $13.4 \%$ increase in hemolysis in G6PD-deficient animals compared to a $2.2 \%$ increase in control animals (Random MO, $\mathrm{p}<0.0001$ ). Methylated spirit exposure produced a $39.7 \%$ increase in hemolysis in G6PD-deficient animals compared to $11.7 \%$ increase in control animals (Random $M O, p<0.0001$ ). In contrast, 

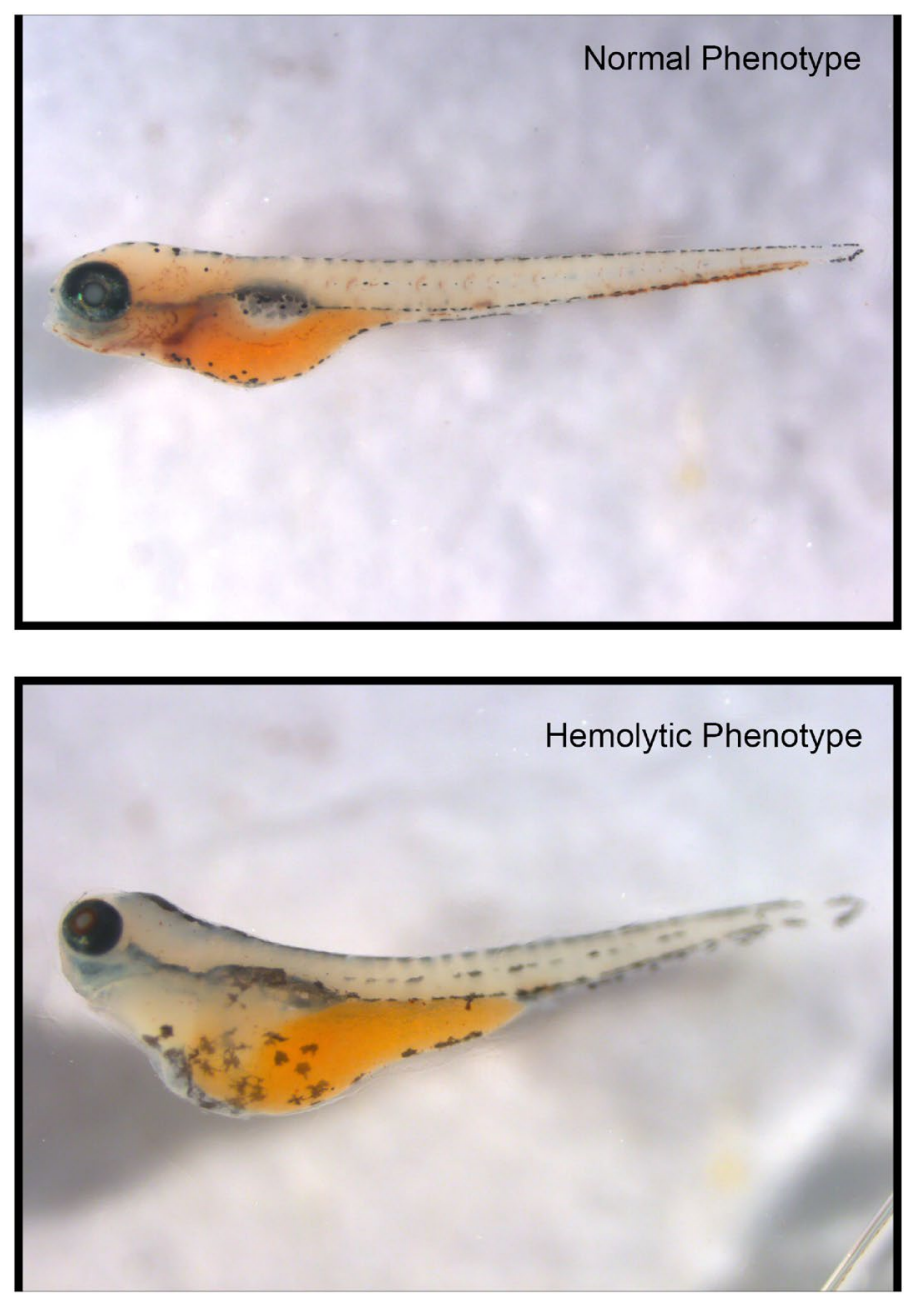

Figure 2. Hemolysis in a G6PD-deficient zebrafish model. Top micrograph shows a zebrafish at $72 \mathrm{hpf}$ with intact red blood cells. Bottom micrograph shows an edematous G6PD-deficient zebrafish at $72 \mathrm{hpf}$ after $48 \mathrm{~h}$ of methylated spirit exposure. Both animals were subject to o-dianisidine staining to show hemoglobin containing red cells. Zebrafish were imaged using a Leica DFC340FX fluorescent microscope with PlanAPO 1.6×/0.05 NA objective. Image capture was performed with Leica Application Suite X 3.6.0.2010 (https://www.leica-microsyste $\mathrm{ms} . c o m /$ products/microscope-software/p/leica-application-suite/).

Yoruba tea exposure produced a $11.0 \%$ increase in hemolytic phenotype in Random morphants and a $15.0 \%$ increase in G6PD morphants, though the difference was not statistically significant $(p=0.42)$.

\section{Discussion}

Experimental results from these experiments indicate that eucalyptus and methylated spirits can trigger hemolytic anemia in the G6PD-deficient model. The use of both of these compounds are commonplace in newborn umbilical cord care, where absorption of the compound is likely. It may be unwise to use these remedies in the newborn given the high rates of G6PD deficiency in Nigeria.

Numerous agents have been shown to cause hemolysis in individuals with G6PD deficiency. Some, such as fava beans, henna, naphthalene, primaquine, dapsone, have conclusively been shown to cause hemolysis and thus should be avoided in individuals with G6PD deficiency ${ }^{1,13,19-21}$. However, in low-resource settings like Nigeria, most individuals do not know their G6PD status. Despite the long-standing recommendation that G6PD screening be done in any country with a G6PD prevalence of more than 3-5\% of males, it is not routinely done in most low- and middle-income countries. Therefore, problematic or potentially problematic agents should be be avoided in all neonates in these countries ${ }^{22}$, and safer treatments should be a promoted.

Parents and clinicians have questioned whether the use of eucalyptus oil and herbs are dangerous in neonates with G6PD deficiency, but little literature exists to answer this important question. Multiple in vitro and in vivo studies suggest that eucalyptus has both antioxidant/anti-inflammatory and pro-oxidant activities. These opposite effects may be due to extraction method, plant source (Eucalyptus is a genus of many species), and the target organ being studied. 


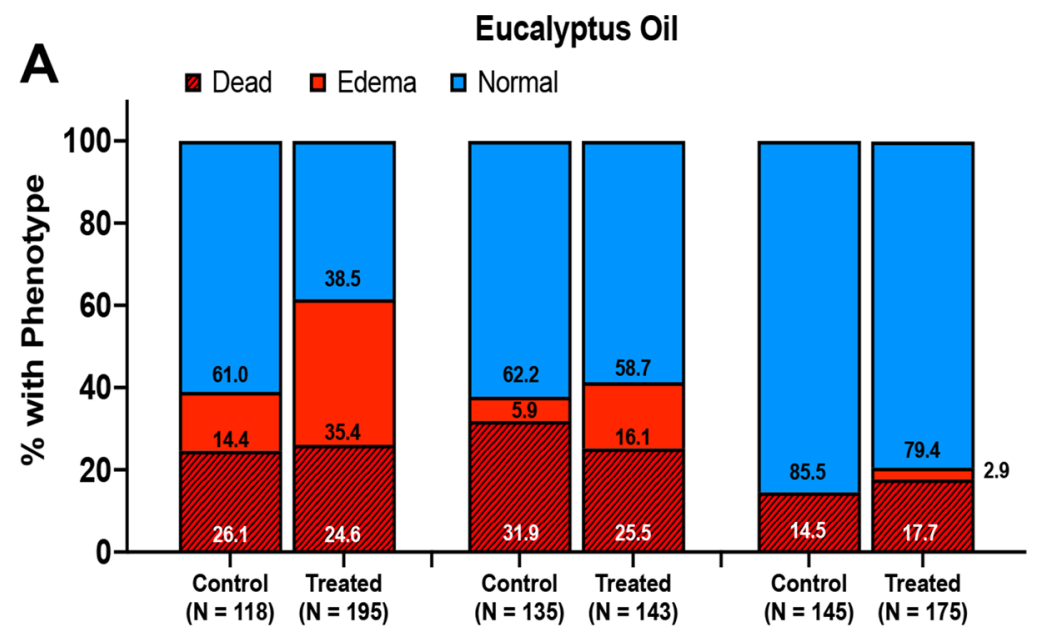

G6PD MO

Random MO

WT

B

Methylated Spirit

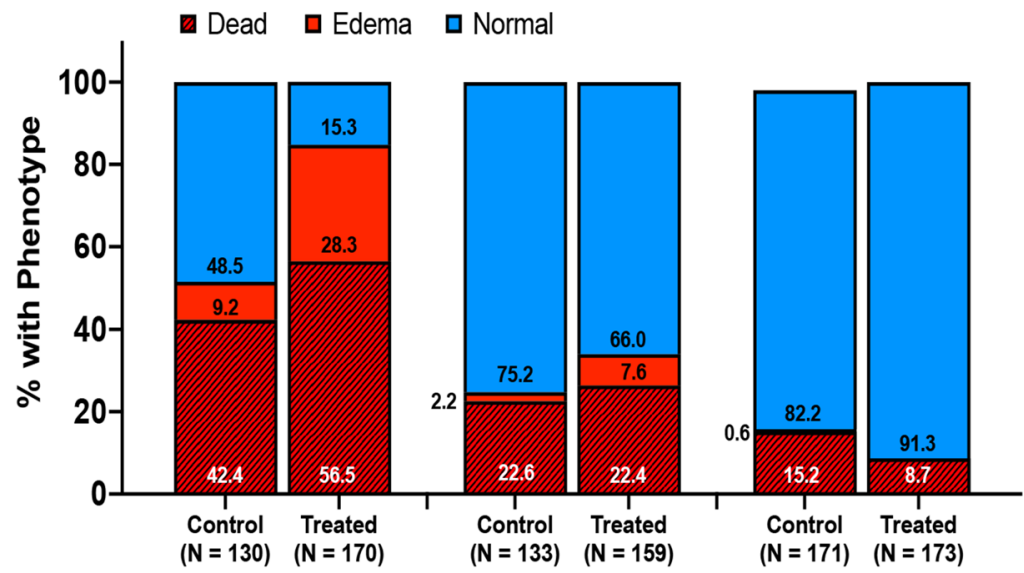

G6PD MO

Random MO

WT

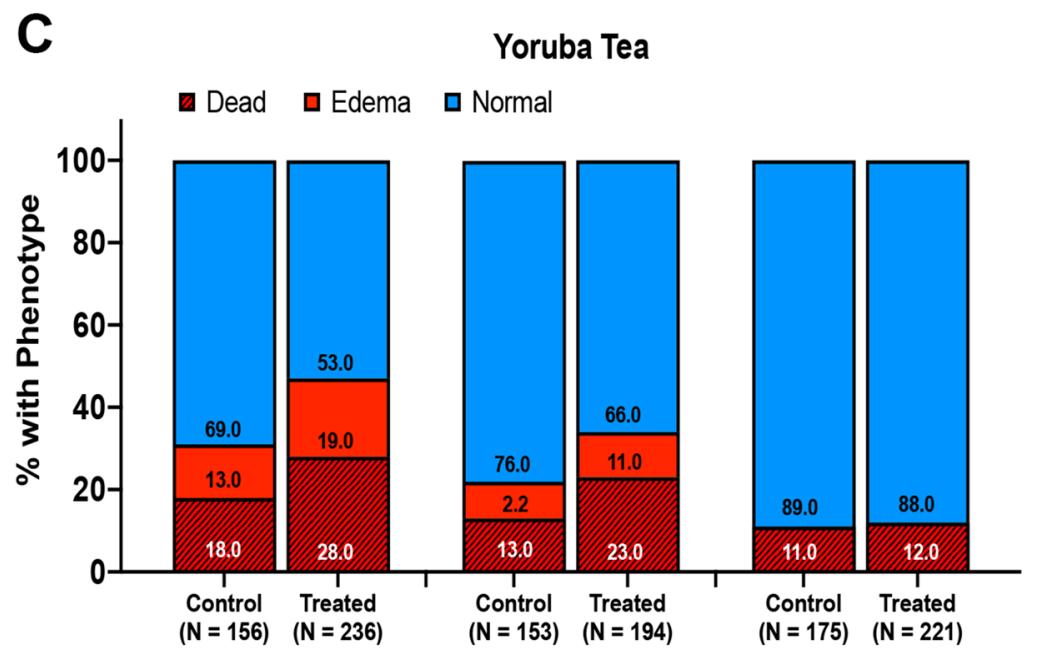

G6PD MO

Random MO

WT

Figure 3. Enumeration of the hemolytic phenotype in G6PD-deficient zebrafish after compound exposure. (A) Impact of eucalyptus oil exposure and regular fishwater on zebrafish in the following groups: G6PD MO, and random MO, uninjected control group. (B) Impact of methylated spirits exposure versus regular fishwater on zebrafish in the following groups: G6PD MO, and random MO, uninjected control group. (C) Impact of Yoruba tea exposure versus regular fishwater on zebrafish in the following groups: G6PD MO, and random MO, uninjected control group. 


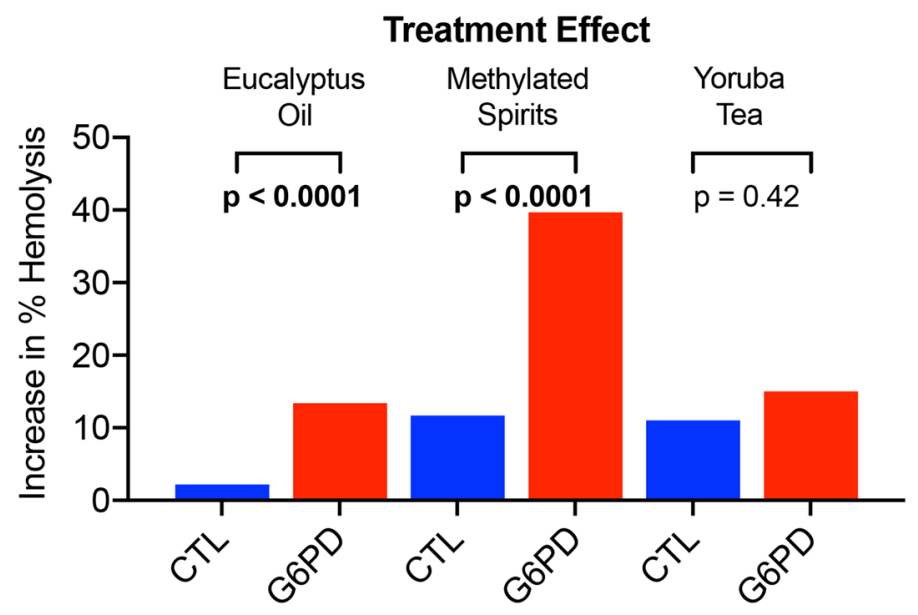

Figure 4. Treatment effect of compounds on G6PD-deficient zebrafish. Tallies were normalized to phenotype data produced in the unexposed embryos to produce a treatment effect. Random MO and G6PD MO injected animals were compared using multivariate analysis was used to separate effects from morpholino exposure versus compound exposure.

However, since severe neonatal hyperbilirubinemia is common in Africa and one of the primary causes is G6PD deficiency, it is crucial to determine which commonly used remedies should be avoided. In our study, zebrafish with G6PD deficiency exposed to eucalyptus oil treatment showed that the treatment triggers hemolytic anemia. These pre-clinical studies this suggest that eucalyptus oil may pose a danger to neonates with G6PD deficiency, but this potential threat must be validated in clinical studies.

Despite the current recommendation by the World Health Organization and others to transition to chlorohexidine for cord care, expense and availability have made this transition slow ${ }^{23}$. As noted in the study by Farouk et al., methylated spirits are also commonly used on the umbilical cords of neonates as a drying agent ${ }^{19}$. Spirits have been presumed to be safer than other native treatments such as plant material because of the risk of neonatal tetanus. However, methylated spirits have not been previously considered by most clinicians to be a risk factor for hemolysis in G6PD-deficient neonates, despite these spirits being a denatured alcohol (ethanol) and a well-described pro-oxidant in several organ systems, including the brain, heart, and liver ${ }^{24-26}$. As with eucalyptus oil, the results from zebrafish with G6PD deficiency exposed to methylated spirits also show a higher rate of hemolysis, suggesting, like eucalyptus, these spirits may be a concern for neonates with G6PD deficiency.

In contrast, diluted Yoruba tea treatment did not trigger hemolytic anemia in the G6PD-deficient zebrafish. This tea is often produced by mothers for cleaning of the umbilical cord as well as for ingestion (by mother and baby). Consequently, it is possible that Yoruba tea treatment does not pose as much risk to neonates who are G6PD-deficient, though it is likely not effective as a cleaning agent. The results of this study are limited by the tea concoction which comes from an assortment of organic compounds that likely vary widely by region. The amounts, concentrations, and treatment of each ingredient are likely also different. We have approximated a "best guess" based on local knowledge by the investigators.

As clinicians and researchers attempt to eliminate acute bilirubin encephalopathy and kernicterus spectrum disorder worldwide, it is essential that agents that are possible triggers for hemolysis in G6PD-deficient neonates be studied both in the lab and in clinical settings. Given the close association of neonatal jaundice and G6PD deficiency throughout much of the world, the results of this study need to be further explored epidemiologically through clinical correlation of exposure histories in infants with G6PD deficiency and jaundice.

\section{Methods}

Zebrafish strains and fish husbandry. Zebrafish (AB strain) were maintained by the University of Minnesota Zebrafish Core Facility. All methods were carried out in accordance with relevant guidelines and regulations. All experimental protocols were according to standardized procedures and approved by the University of Minnesota's International Animal Care and Use Committee. Wild type fish were obtained from Segrest Farms (Gibsonton, FL, USA) and bred in-house.

Morpholinos. The G6PD antisense translation blocker and splice morpholino's (MO) were designed and purchased from Gene Tools (Philomath, OR, USA) and used as previously described ${ }^{18}$. The MOs were dissolved in water at a stock concentration of $8 \mathrm{mg} / \mathrm{mL} .3 \mathrm{~nL}$ volumes of $\mathrm{MO}$ were delivered via air pressure injection into one- to two-cell stage embryos. The G6PD MOs were combined in a 1:1 ratio for delivery of 1.2 pmol. Random MOs at $1.2 \mathrm{pmol}$ were used for control injection. The G6PD translation blocking the MO sequence was 5'-gcgctcgctcgactgcccatcattt-3'; the splice-blocking MO sequence was 5'-ataataaaagacttaccgaagcgccc-3'. The random MO was created with a similar GC content and random bases used. Random MOs from two different batches were used. Wildtype (WT) embryos were kept from each clutch as a non-injected control, a signal of the clutch's overall health before treatment. 
Prooxidant exposure. All zebrafish embryos were dechorionated at $24 \mathrm{~h}$ post-fertilization (hpf) and placed in 6- or 12-well plates for treatment, depending on the size of the clutch. All chemicals were dissolved in zebrafish water ("fishwater", $0.006 \%$ Instant Ocean in MilliQ water) prior to treatment. For each chemical, dose curves were established to determine which concentrations yielded the best survival rate for uninjected embryos. Final dosages varied between chemicals. Silverbird Eucalyptus Oil from Bell's Healthcare in the United Kingdom was dissolved at a ratio of 1:5000 in fishwater. Methylated Spirits from Aicon Pharmaceuticals LTD was dissolved at a ratio of 1:5000 in fishwater. A Yoruba tea concoction consisting of various roots, leaves, bark, and herbs obtained from a shaman in Nigeria and boiled in fishwater for $20 \mathrm{~min}$, was dissolved at a ratio of 1:75 in fishwater. Fresh dilutions were made for each new clutch, with treatment lasting until 72 hpf as previously described ${ }^{18}$. In each experiment, half of the embryos were subjected to treatment while the other half was maintained in fishwater as a control. Treated and untreated embryos were kept in separate incubators to avoid any possible vapor-related contamination from evaporated chemicals. After $48 \mathrm{~h}$ of compound exposure, animals were tallied according to phenotype. Chi-square analyses were performed to evaluate differences between wildtype and edema phenotypes. Multivariate analysis was used to separate effects from morpholino exposure versus compound exposure. Statistical software used for analyzing the data was JMP version 14.0.

Staining and imaging. Hemoglobin staining was performed as described previously ${ }^{27}$. Dechorionated live embryos at $72 \mathrm{hpf}$ were stained in $0.6 \mathrm{mg} / \mathrm{mL}$ o-dianisidine (Sigma-Aldrich, St. Louis, MO) containing $0.01 \mathrm{~mol} / \mathrm{L}$ sodium acetate $(\mathrm{pH} 4.5), 0.65 \% \mathrm{H}_{2} \mathrm{O}_{2}$, and $40 \%$ ethanol in the dark for $30 \mathrm{~min}$. Embryos were then dehydrated through graded ethanol washes of $50 \%, 75 \%$, and $100 \%$, for 5 min each. Finally, embryos were cleared in benzyl benzoate and benzyl alcohol at a 2:1 ratio. Embryos were stored in the clearing solution until imaged using a Leica DFC340FX fluorescent microscope with PlanAPO 1.6×/0.05 NA objective (Leica Camera Inc., Allendale, NJ, USA). Image capture was done with Leica LAS software, and post processing was done using Adobe Photoshop CS4 (Mountain View, CA, USA).

\section{Data availability}

The datasets generated during and/or analyzed during the current study are available from the corresponding author on reasonable request.

Received: 1 March 2020; Accepted: 6 October 2020

Published online: 05 November 2020

\section{References}

1. Ezeaka, C. V., Ugwu, R. O., Mukhtar-Yola, M., Ekure, E. N. \& Olusanya, B. O. Pattern and predictors of maternal care-seeking practices for severe neonatal jaundice in Nigeria: a multi-centre survey. BMC Health Serv. Res. 14, 192. https://doi.org/10.1186/14726963-14-192 (2014).

2. Shewamene, Z., Dune, T. \& Smith, C. A. The use of traditional medicine in maternity care among African women in Africa and the diaspora: a systematic review. BMC Complement. Altern. Med. 17, 382. https://doi.org/10.1186/s12906-017-1886-x (2017).

3. Roll Back Malaria: spotlight on Africa. TDR News 10, 15 (2000).

4. Farouk, Z. L., Slusher, T. M., Danzomo, A. A. \& Slusher, I. L. Factors influencing neonatal practice in a rural community in Kano (Northern), Nigeria. J. Trop. Pediatr. https://doi.org/10.1093/tropej/fmz012 (2019).

5. Guindo, A., Fairhurst, R. M., Doumbo, O. K., Wellems, T. E. \& Diallo, D. A. X-linked G6PD deficiency protects hemizygous males but not heterozygous females against severe malaria. PLoS Med. 4, e66. https://doi.org/10.1371/journal.pmed.0040066 (2007).

6. Clark, T. G. et al. Allelic heterogeneity of G6PD deficiency in West Africa and severe malaria susceptibility. Eur. J. Hum. Genet. 17, 1080-1085. https://doi.org/10.1038/ejhg.2009.8 (2009).

7. Manjurano, A. et al. African glucose-6-phosphate dehydrogenase alleles associated with protection from severe malaria in heterozygous females in Tanzania. PLoS Genet. 11, e1004960. https://doi.org/10.1371/journal.pgen.1004960 (2015).

8. Beutler, E. et al. International Committee for Standardization in Haematology: recommended screening test for glucose-6-phosphate dehydrogenase (G-6-PD) deficiency. Br. J. Haematol. 43, 465-467 (1979).

9. Bernstein, S. C., Bowman, J. E. \& Kaptue Noche, L. Population studies in Cameroon: hemoglobin S, glucose-6-phosphate dehydrogenase deficiency and falciparum malaria. Hum. Hered. 30, 251-258 (1980).

10. Tan, K. L. Glucose-6-phosphate dehydrogenase status and neonatal jaundice. Arch. Dis. Child 56, 874-877 (1981).

11. Lauden, S. M. et al. Prevalence of glucose-6-phosphate dehydrogenase deficiency in Cameroonian blood donors. BMC Res. Notes 12, 195. https://doi.org/10.1186/s13104-019-4226-z (2019).

12. Williams, O. et al. Glucose-6-phosphate dehydrogenase deficiency in Nigerian children. PLoS ONE 8, e68800. https://doi. org/10.1371/journal.pone.0068800 (2013).

13. Lee, S. W., Lai, N. M., Chaiyakunapruk, N. \& Chong, D. W. Adverse effects of herbal or dietary supplements in G6PD deficiency: a systematic review. Br. J. Clin. Pharmacol. 83, 172-179. https://doi.org/10.1111/bcp.12976 (2017).

14. Beutler, E. Glucose-6-phosphate dehydrogenase deficiency: a historical perspective. Blood 111, 16-24. https://doi.org/10.1182/ blood-2007-04-077412 (2008).

15. Cappellini, M. D. \& Fiorelli, G. Glucose-6-phosphate dehydrogenase deficiency. Lancet 371, 64-74. https://doi.org/10.1016/s0140 $-6736(08) 60073-2(2008)$

16. Nkhoma, E. T., Poole, C., Vannappagari, V., Hall, S. A. \& Beutler, E. The global prevalence of glucose-6-phosphate dehydrogenase deficiency: a systematic review and meta-analysis. Blood Cells Mol. Dis. 42, 267-278. https://doi.org/10.1016/j.bcmd.2008.12.005 (2009).

17. Olusanya, B. O., Osibanjo, F. B., Mabogunje, C. A., Slusher, T. M. \& Olowe, S. A. The burden and management of neonatal jaundice in Nigeria: a scoping review of the literature. Niger. J. Clin. Pract. 19, 1-17. https://doi.org/10.4103/1119-3077.173703 (2016).

18. Patrinostro, X., Carter, M. L., Kramer, A. C. \& Lund, T. C. A model of glucose-6-phosphate dehydrogenase deficiency in the zebrafish. Exp. Hematol. 41, 697-710 e692. https://doi.org/10.1016/j.exphem.2013.04.002 (2013).

19. Farouk, Z. L., Ibrahim, M. \& Ogala, W. N. Glucose-6-phosphate dehydrogenase deficiency; the single most important cause of neonatal hyperbilirubinemia in Kano, Nigeria. Niger. J. Paediatr. 22, 44-49 (2017).

20. Belfield, K. D. \& Tichy, E. M. Review and drug therapy implications of glucose-6-phosphate dehydrogenase deficiency. Am. J. Health Syst. Pharm. 75, 97-104. https://doi.org/10.2146/ajhp160961 (2018). 
21. Lee, S. W., Chaiyakunapruk, N. \& Lai, N. M. What G6PD-deficient individuals should really avoid. Br. J. Clin. Pharmacol. 83, 211-212. https://doi.org/10.1111/bcp.13091 (2017).

22. WHO Working Group. Glucose-6-phosphate dehydrogenase deficiency. Bull. World Health Organ. 67, 601-611 (1989).

23. Hill, S. R. Putting the priorities first: medicines for maternal and child health. Bull. World Health Organ. 90, 236-238. https://doi. org/10.2471/BLT.11.088658 (2012).

24. Cederbaum, A. I., Lu, Y. \& Wu, D. Role of oxidative stress in alcohol-induced liver injury. Arch. Toxicol. 83, 519-548. https://doi. org/10.1007/s00204-009-0432-0 (2009).

25. Luo, J. Mechanisms of ethanol-induced death of cerebellar granule cells. Cerebellum 11, 145-154. https://doi.org/10.1007/s1231 1-010-0219-0 (2012).

26. Mustroph, J., Lebek, S., Maier, L. S. \& Neef, S. Mechanisms of cardiac ethanol toxicity and novel treatment options. Pharmacol. Ther. 197, 1-10. https://doi.org/10.1016/j.pharmthera.2018.12.006 (2019).

27. Detrich, H. W. 3rd. et al. Intraembryonic hematopoietic cell migration during vertebrate development. Proc. Natl. Acad. Sci. U.S.A. 92, 10713-10717. https://doi.org/10.1073/pnas.92.23.10713 (1995).

\section{Author contributions}

T.L., T.S., A.O. authored the manuscript and interpreted the data. M.S., A.E., J.W. performed the injections, staining and enumerated animals. Z.F., T.S., S.C. performed manuscript editing. All authors reviewed the manuscript.

\section{Competing interests}

The authors declare no competing interests.

\section{Additional information}

Supplementary information is available for this paper at https://doi.org/10.1038/s41598-020-75823-x.

Correspondence and requests for materials should be addressed to T.L.

Reprints and permissions information is available at www.nature.com/reprints.

Publisher's note Springer Nature remains neutral with regard to jurisdictional claims in published maps and institutional affiliations.

(c) (i) Open Access This article is licensed under a Creative Commons Attribution 4.0 International

License, which permits use, sharing, adaptation, distribution and reproduction in any medium or format, as long as you give appropriate credit to the original author(s) and the source, provide a link to the Creative Commons licence, and indicate if changes were made. The images or other third party material in this article are included in the article's Creative Commons licence, unless indicated otherwise in a credit line to the material. If material is not included in the article's Creative Commons licence and your intended use is not permitted by statutory regulation or exceeds the permitted use, you will need to obtain permission directly from the copyright holder. To view a copy of this licence, visit http://creativecommons.org/licenses/by/4.0/.

(C) The Author(s) 2020 